Exp Brain Res (1990) 79:192-206

\title{
Neural control of vocalization in bats: mapping of brainstem areas with electrical microstimulation eliciting species-specific echolocation calls in the rufous horseshoe bat
}

\author{
G. Schuller and S. Radtke-Schuller \\ Zoologisches Institut der Ludwig-Maximilians-Universität, Luisenstrasse 14, D-8000 München 2, Federal Republic of Germany
}

Summary. 1. The functional role of brainstem structures in the emission of echolocation calls was investigated in the rufous horseshoe bat, Rhinolophus rouxi, with electrical low-current microstimulation procedures. 2. Vocalizations without temporal and/or spectral distortions could be consistently elicited at low threshold currents (typically below $10 \mu \mathrm{A}$ ) within three clearly circumscribed brainstem areas, namely, the deep layers and ventral parts of the intermediate layers of the superior colliculus (SC), the deep mesencephalic nucleus (NMP) in the dorsal and lateral midbrain reticular formation and in a distinct area medial to the rostral parts of the dorsal nucleus of the lateral lemniscus. The mean latencies in the three vocal areas between the start of the electrical stimulus and the elicited vocalizations were $47 \mathrm{msec}, 38 \mathrm{msec}$ and $31 \mathrm{msec}$, respectively. 3 . In pontine regions and the cuneiform nucleus adjacent to these three vocal areas, thresholds for eliciting vocalizations were also low, but the vocalizations showed temporal and/or spectral distortions and were often accompanied or followed by arousal of the animal. 4. Stimulus intensity systematically influenced vocalization parameters at only a few brain sites. In the caudo-ventral portions of the deep superior

\footnotetext{
Offprint requests to: G. Schuller (address see above) Abbreviations: $\mathrm{AP}=$ pretectal area; $\mathrm{BIC}=$ brachium of the inferior colliculus: $\mathrm{CM}=$ mamillary body: $\mathrm{CTm}=$ medial trapezoid body: $\mathrm{CUN}=$ cuneiform $\mathrm{ncl}$; $\mathrm{HYP}=$ hypothalamus; IC $(\mathrm{rp})=$ inferior colliculus (rostral pole); IP = interpeduncular ncl.; $L L(v, i, d)=$ lateral lemniscus (ventral, intermediate, dorsal): $\mathrm{MGB}=$ medial geniculate body; $\mathrm{NMP}=$ deep mesencephalic nucleus: $\mathrm{NR}=$ red nucleus; $\mathrm{P}=$ pons; $\mathrm{PAG}=$ periaqueductal gray; $\mathrm{PC}=$ cerebral peduncle; $\mathrm{PO}=$ pons oralis; $\mathrm{RD}=$ raphe dorsalis; $\mathrm{RRF}=$ retrorubral field; $\mathrm{RTP}=$ ncl. reticularis pontis: $\mathrm{SC}(\mathrm{s}, \mathrm{i}, \mathrm{d})=$ superior colliculus (superficial, intermediate, deep); $\mathrm{SG}=$ suprageniculate ncl.; $\mathrm{SN}=$ substantia nigra; $3=$ ncl. of the oculomotor nerve
}

colliculus the sound pressure level of the vocalizations systematically increased with stimulus intensity. Bursts of multiple vocalizations were induced at locations ventral to the rostral parts of the cuneiform nucleus. No stimulus-intensity dependent frequency changes of the emitted vocalizations were observed. 5. The respiratory cycle was synchronized to the electrical stimuli in all regions where vocalizations could be elicited as well as in more ventrally and medially adjacent areas not yielding vocalizations on stimulation. 6 . The possible functional involvement of the "vocal" structures in the audio-vocal feedback system of the Dopplercompensating horseshoe bat is discussed.

Key words: Vocalization - Microstimulation Mapping - Brainstem - Bat

\section{Introduction}

As in many mammals, echolocating bats use vocalizations for intraspecific communication. However, such bats are mainly noted for production of sonar signals whose echoes act as acoustical carriers of target information for orientation and prey capture. Both echolocation signals and communication signals are produced by the larynx and they presumably share the same neuronal control centers. In higher mammals, including humans, articulation is the most important shaping mechanism for vocalizations (Ploog 1988), but in lower mammals the acoustical properties of vocalizations are governed by direct control of the larynx, and active shaping by the vocal tract is very limited.

Since sound production in mammals is a complex coordination of several motor acts involving the control of the laryngeal muscles, the respiratory 
muscles and, the articulary muscles of throat, mouth and nose (Thoms and Jürgens 1987), we might not expect a simple neural representation for the isolated vocalization functions. Rather, there may be a multimodal representation of vocal functions coordinated by different brain areas. At peripheral levels, the selective denervation of the larynx has shown the functional importance of the different laryngeal muscles for the spectral composition of the vocalizations : the superior laryngeal nerve (motor branch) controls predominantly the frequency of the vocalizations and the recurrent (or inferior) laryngeal nerve influences mainly the temporal parameters of vocalizations (Schuller and Suga 1976; Jürgens et al. 1978, Schuller and Rübsamen 1981; Rübsamen and Schuller 1981). As determined by tracer studies, the motoneurons of laryngeal innervation are located in the ncl. ambiguus and show an orderly arrangement for the different laryngeal nerves: The motoneurons of the superior laryngeal nerve are located more rostrally within the ncl. ambiguus than those of the recurrent laryngeal nerve (Schweizer et al. 1981; Rübsamen and Betz 1986; Thoms and Jürgens 1981).

Potential sources of input to the ncl. ambiguus have been revealed mainly by electrical stimulation (e.g. Novick and Griffin 1961; Suthers and Fattu 1982; Suga et al. 1973; Jürgens 1976; Jürgens and Pratt 1979) or chemical stimulation (Richter and Jürgens 1986; Jürgens and Richter 1986) to determine brain areas where species-specific vocalizations can be elicited. It is only in primates that the brain structures involved in sound production have been investigated at all levels of the central nervous system (Jürgens 1988). The stimulation studies together with lesion and tracer studies have led to a concept of hierarchical organization of the efferent vocalization system (Jürgens 1988; MüllerPreuss and Ploog 1983). An important role is attributed to the central gray of the midbrain and structures of the adjacent reticular formation as centers where all information from higher levels (e.g. hypothalamus, gyrus cinguli) is integrated and transmitted to the nucleus ambiguus (Jürgens and Pratt 1979). Kirzinger and Jürgens (1985) demonstrated that ablation of brain structures above the level of the periaqueductal gray (PAG) did not alter the spectral composition of spontaneously emitted calls, suggesting that motor coordination of laryngeal muscles is controlled below the mesencephalic level.

Current evidence suggests that bats have a similar organization of the vocalization system, and electrical stimulation in different species (Novick and Griffin 1961; Suthers and Fattu 1982; Suga et al. 1973; Gooler and O'Neill 1987) yielded emission of species-specific vocalizations at comparable stimulation sites in the forebrain and the brainstem. Since accurate histological localization of stimulation sites is often lacking in these investigations, the exact anatomical identification of stimulated brain structures is difficult. Thus, for example, it cannot be decided whether the effective stimulation sites for eliciting vocalizations in midbrain areas were in the periaqueductal gray proper or in neighboring brain structures. Tracer injections into physiologically described regions of the ncl. ambiguus and the adjacent reticular formation of Rhinolophus rouxi have disclosed a variety of projections to the nucleus ambiguus possibly relevant for vocal control (Rübsamen and Schweizer 1986). The ncl. ambiguus receives input not only from the lateral parts of the PAG but also from the cuneiform nucleus (CUN), the deep layers of the superior colliculus (SC) and pontine nuclei. Since the nucleus ambiguus is not exclusively involved in the control of laryngeal muscles, functional interpretation of the different projections for vocal control is not straightforward, and requires verification of each projection by physiological and anatomical methods.

The control of frequency, harmonic composition, intensity, time course of modulations and duration of the echolocation calls is highly important for echolocating bats in order to meet the requirements of specific orientation tasks. Therefore it might be expected that the spectro-temporal parameters of vocalizations could be modified by electrical stimulation of vocal control centers. To date only Gooler and O'Neill (1987) have demonstrated a systematic dependence of the emitted frequency of orientation calls, in the bat Pteronotus p. parnelli, on the location of the electrical stimulation site within the anterior cingulate cortex. Other correlations between specific parameters of the spectro-temporal composition of elicited echolocation calls and parameters of the electrical stimuli have not systematically been demonstrated.

The bat Rhinolophus rouxi emits long, constant frequency calls of 10 to $100 \mathrm{~ms}$ duration at about $78 \mathrm{kHz}$ preceded and followed by frequency modulations (Schuller 1980). During flight the bat lowers the emitted frequency in order to compensate for the Doppler-shifts introduced by the relative speed between bat and target (Schnitzler 1968; Schuller et al. 1974). The control system for Doppler-shift compensation behaviour uses auditory feedback in order to adjust the emitted frequency. Thus, this species is an ideal animal model for studying the vocal control of emitted frequency as 
an isolated parameter which can be experimentally influenced via auditory feedback.

The object of the present experiments was to define accurately the anatomical structures involved in vocal control of echolocation call emission, and to shed light on their functional role for the formation of vocal patterns. To this purpose brainstem regions of the horseshoe bat were systematically scanned with electrical stimulation, and the sites for eliciting echolocation calls were carefully reconstructed. The influence of electrical stimulation on the respiratory pattern in conjunction with elicited vocalization is also described.

\section{Material and methods}

Eighteen rufous horseshoe bats, Rhinolophus rouxi, were used in these studies. Each bat was kept in captivity under seminatural conditions for under a year.

The animals were surgically prepared under Halothane anesthesia. The skin overlying the skull was cut and reflected to the sides in order to afix, with dental cement, a tube that was attached to the stereotaxic device during experiments. Tissue was carefully cleaned from an area of skull about $1.5 \mathrm{~mm}$ left and right of midline. This area was used to determine the position of the skull in stereotaxic coordinates and to place holes for electrodes. The animals were then allowed to recover from surgery overnight. Throughout the experiments, the wound margins were treated with local anesthetics (Novocain). The experiments were conducted in an acoustic chamber with foamcovered walls to reduce the reflection of ultrasonic vocalizations. The animals were placed in a holder which prevented gross body movements, and the head was immobilized by attaching the surgically-afixed tube to the head-holder that allowed highly accurate repositioning (about $10 \mu \mathrm{m}$ ) of the animal in the stereotaxic device. The stereotaxic device and the procedures to accurately determine the skull position in stereotaxic coordinates are described elsewhere (Schuller et al. 1986). The method yields a typical accuracy for the localization of stimulation sites of $100 \mu \mathrm{m}$ in all 3 dimensions. The localization of stimulus sites within the brain was further verified by injection of tracer substances, such as HRP or WGA conjugated with HRP, or by placing small electrolytic lesions at the end of the experiment.

The measurements for determining the skull position in stereotaxic coordinates were done during a short (about 1 hour) session at the first postoperative day. The stimulation experiments started at the second postoperative day with daily sessions of maximally six hours and could be repeated for up to three weeks. To prepare the animal for electrical stimulation, a hole wals cut into the skull over the target area and the dura was perforated under local anesthesia. The holes had diameters typically below $500 \mu \mathrm{m}$ and several electrode penetrations with different rostro-caudal or medio-lateral inclinations were conducted through each hole. The positions of the penetrations were all referred to a common reference point and subsequently reconstructed computationally. The reconstruction yielded the spatial distribution of stimulation sites in coordinates of a brain atlas (S. Radtke-Schuller, unpublished).

Parylene-coated tungsten electrodes (Micro Probe Inc.) with impedances between 0.5 and $2 \mathrm{MOhm}$ were lowered from the surface of the brain in steps of $100 \mu \mathrm{m}$ with a piezoelectric stepper (Burleigh Inchworm). The electrode tips had diameters of $2-3 \mu \mathrm{m}$ and were uninsulated for $5-10 \mu \mathrm{m}$. An indifferent clectrode contacting the dura under the anterior part of the skull was chronically implanted.

Electrical stimuli were produced with a Grass stimulator (Type S88B) and transmitted to the electrode with a Grass isolation unit (PSIU 6) with negative polarity. The stimuli consisted of trains of rectangular pulses of $100 \mu \mathrm{sec}$ duration at a frequency of $1 \mathrm{kHz}$. The trains were generally $15 \mathrm{msec}$ long and presented at repetition rates between 2 and $15 \mathrm{~Hz}$. The stimulus currents typically ranged from 1 to $50 \mu \mathrm{A}$, and in a few cases up to $150 \mu \mathrm{A}$.

In order to avoid stimulation leading to general arousal of the animal, the bats were visually monitored during experiments with a color TV-camera (Hitachi HV 9017). Oro-facial responses and ear movements to selected sequences of stimulus trains were recorded on a video recorder (Panasonic NV 8200) for further evaluation.

Vocalizations of the bats were monitored with a Bruel \& Kjaer 1/4" ultrasonic microphone (type 4135), amplified, and then fed to both a frequency-to-voltage converter and an AC-toDC converter (Phillips PM 5171). The frequency of vocalization it as measured by a frequency-to-voltage converter with a resolution better than $50 \mathrm{~Hz}$. The intensity of vocalization was determined from an AC/DC converter set in logarithmic scale. The latency of the call from the start of the electrical stimulus, and the duration of the call, were monitored on the oscilloscope.

The respiratory flow was monitored with a thermistor directly in front of the bat's nostril. The diameter of the thermistor was only $0.4 \mathrm{~mm}$ and did not obstruct or distort the vocalizations enitted through the nostrils. The frequency and intensity signals were recorded on FM-channels of a Nagra TI instrumentation tape recorder (Kudelski, Geneva), and the respiration signal and the electrical stimuli were fed to direct channels of the recorder. The electrical stimulus and the frequency-to-voltage converter output signal were also recorded on the acoustic channels of the video recorder in order to analyze the synchrony of vocal and facial motor activities during sound emission.

The threshold for electrically stimulated vocalizations or related oro-facial movements was visually determined on the oscilloscope and the TV-screen, respectively, as the stimulus intensity was gradually increased.

The frequency and amplitude of the vocalizations, the respiration signal and the electrical stimuli were recorded for typical stimulation sequences at stimulation sites that yielded vocalizations. After the experiment, the taped signals were plotted on a 4-channel high speed recorder (Watanabe Mark VII) at reduced speed and then analyzed for their magnitude, duration and temporal relationship as well as for their spectral similarity to natural echolocation calls.

Since the location of stimulation sites could be computationally transformed into coordinates of a brain atlas of $R$ hinolophus rouxi (S. Radtke-Schuller, unpublished), the data from all penetration angles were pooled into a common data base. The characteristics of each stimulation site, e.g.. threshold for different responses, were entered together with the coordinates into a data base program (Reflex, Borland) and the brain sites plotted for different selections of stimulation parameters and response characteristics.

\section{Results}

Electrically stimulated vocalizations were obtained from the brainstems and midbrains of 18 adult bats, Rhinolophus rouxi. Depending on the stimulation site the thresholds for eliciting vocalizations, the parameters of the vocalizations and the accom- 
panying motor patterns of mouth/nose, ear and body movements differed considerably.

At stimulation sites specific for the production of undistorted echolocation calls a similar sequence of changes in response to increasing stimulus currents was typical. At threshold currents, elicited vocalizations were accompanied by synchronized expiratory activity and often by coordinated ear movements. Increasing the stimulating current generally improved the synchrony of the vocalization to the stimulus and induced, if not yet present, coordinated ear movements and/or oro-facial movements. Relatively high stimulating currents produced body movements, and the highest stimulation currents (which were generally avoided) led to general arousal of the animal. Stimulation currents were kept as low as possible, typically in a range between 1 and $50 \mu \mathrm{A}$. To maintain the specificity of stimulation site, currents never exceeded $150 \mu \mathrm{A}$.

\section{Functional mapping of brainstem and midbrain areas}

Mapping of brain areas which elicited vocalizations was facilitated by a computer-aided reconstruction of the stimulation loci, which were verified with electrolytic lesions or focal tracer injections. The typical absolute spatial accuracy of stimulation sites in atlas coordinates was better than $100 \mu \mathrm{m}$ along all three dimensions.

The criteria used to determine whether a particular brain region was "specific" for the production of biosonar vocalizations were:

1. the threshold currents for triggering vocalization were below $10 \mu \mathrm{A}$; these low currents guaranteed a sufficiently fine spatial resolution (effective stimulation within a radius of approximately $80 \mu \mathrm{m}) ; 2$. the vocalizations corresponded to spontaneously produced echolocation calls with respect to their spectral and temporal pattern; 3. no body movements other then ear, mouth, or nose. accompanied the vocalizations; 4 . the latency between the onset of a stimulus train and the elicited vocalization was stable and shorter than $100 \mathrm{msec}$; and 5 . vocalizations were not uttered as a consequence of stimulus-induced arousal of the bat.

Figure 1 shows the stimulation sites (small circles) where thresholds for eliciting vocalizations were below $10 \mu \mathrm{A}$. Each frontal brain section, spaced $264 \mu \mathrm{m}$ apart, contains stimulation sites pooled over $264 \mu \mathrm{m}( \pm 132 \mu \mathrm{m})$ in the rostrocaudal dimension (slice \#253 through \# 377). The total area that was probed with electrical stimuli is outlined by heavy lines in each section. Within these

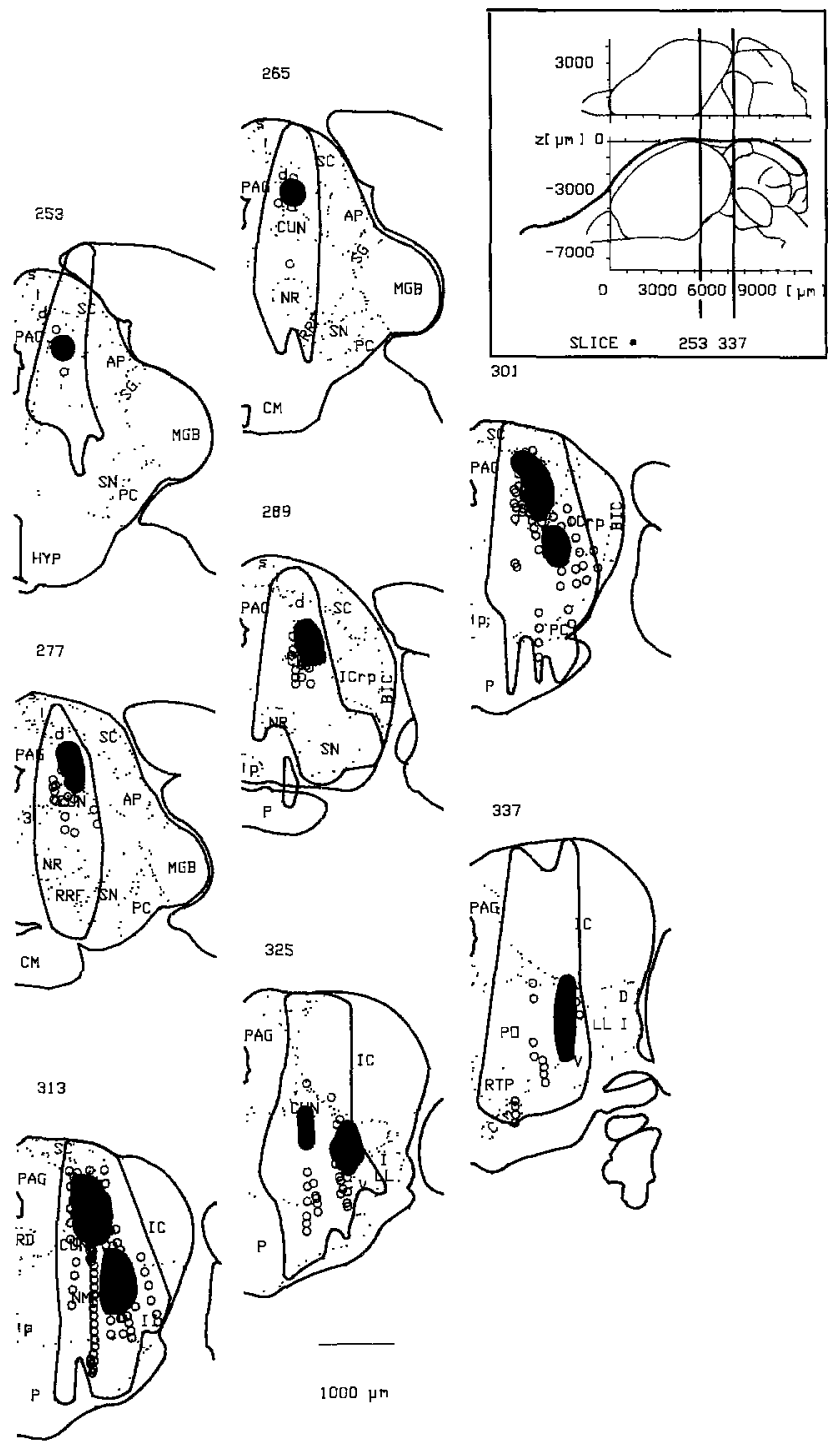

Fig. 1. Local distribution of brain sites yielding vocalizations with electrical microstimulation. The data are represented in 8 consecutive frontal sections on which the results are pooled over a rostro-caudal extent of $264 \mu \mathrm{m}$. The inset shows the level of the most rostral and most caudal section (vertical lines) in a sagittal view of the brain. Brain areas which have been systematically probed with electrical stimulation for eliciting echolocation calls are surrounded by heavy lines within the outlines of the sections. In these areas the stimulation loci were spaced less than $200 \mu \mathrm{m}$ apart. The brain sites where vocalization could be elicited at stimulus currents below $10 \mu \mathrm{A}$ are represented as circles or by the filled areas within which the vocalization was optimally and most consistently evoked, i.e. at lowest thresholds, at consistent latencies, without distortions and without any accompanying body movement or arousal. The main brain areas for electrically eliciting vocalizations under optimal conditions are the ventral parts of the intermediate layers and the deep layers of the SC (\#253-313), an area in the dorsal reticular formation (deep mesencephalic nucleus, NMP, \#301.313) and an area medial to the most rostral parts of the dorsal nucleus of the lateral lemniscus (\#325-337) 

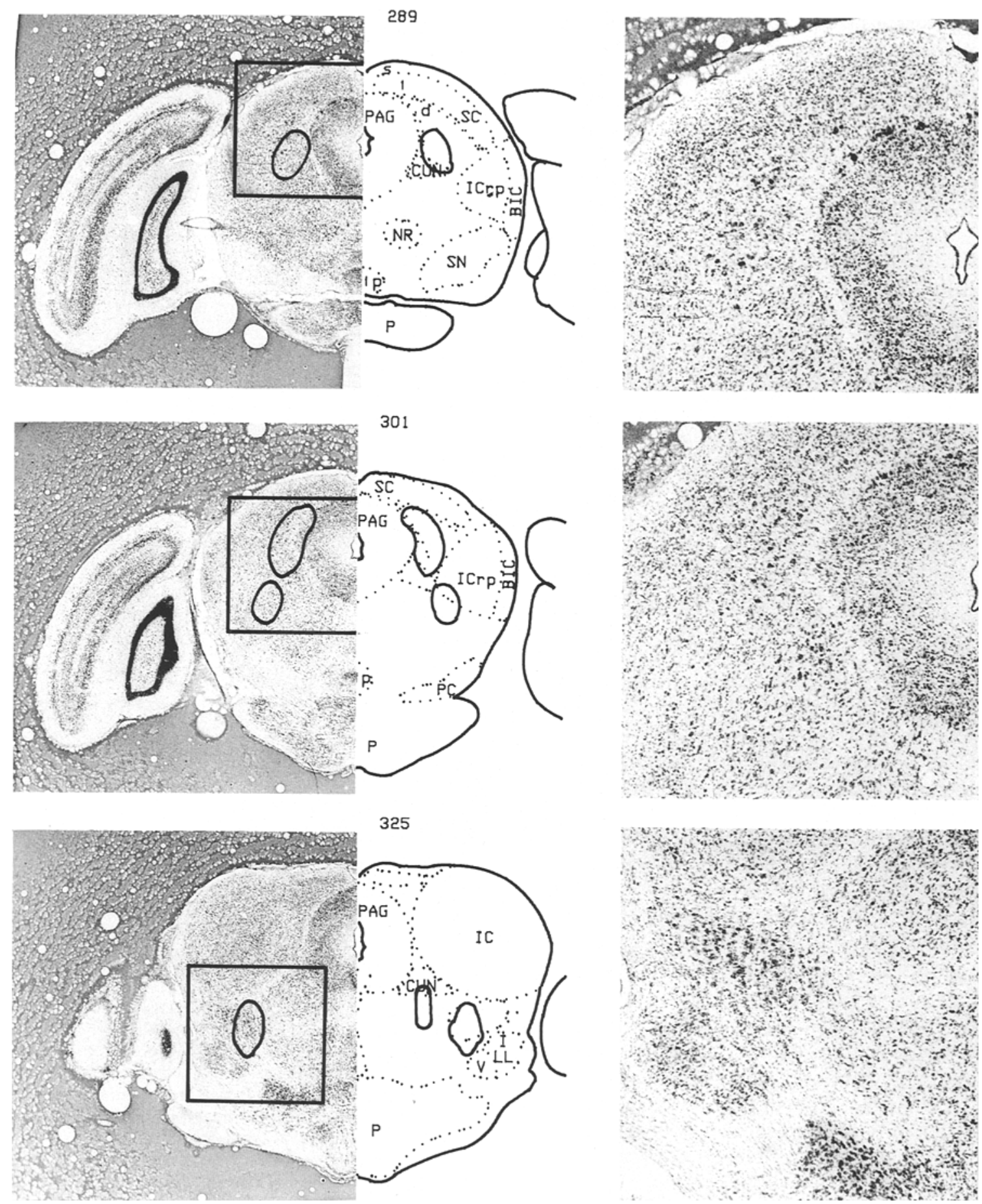

$1000 \mu \mathrm{m}$

Fig. 2. Optimal stimulation sites at 3 rostrocaudal levels with the corresponding Nissl sections showing the corresponding anatomical structures in the bat. The magnified photograph shows details of the respective brain area. A: level of the SC (\# 289), B: NMP (\#301) and $C$ : area medial and rostral to the DNLL (\#325) 
areas the stimulation sites were never more distant from each other than $200 \mu \mathrm{m}$. Only the very dorsolateral portion and the areas near the mid sagittal plane were not tested by electrical stimulation.

Three distinct brain areas fulfilled all of the above criteria to be considered vocalization centers and are represented in figure 1 as filled areas. These were 1 . the deep layers and ventral parts of the intermediate layers of the SC (SCd, SCi), 2. an area in the dorsolateral part of the midbrain reticular formation, possibly the deep mesencephalic nucleus (NMP), and 3 . a cell aggregation of mainly large neurons located medial to the rostral part of the dorsal nucleus of the lateral lemniscus (DNLL).

Stimulation sites in the deep layers of the SC covered a relatively large rostro-caudal extent of about $1320 \mu \mathrm{m}$ and only slightly invaded the neighboring PAG of the midbrain. Fig. 2A represents the optimal stimulation sites at the rostrocaudal level of slice \#289 superimposed to the corresponding Nissl-stained sections.

The two other brain areas in which vocalization could specifically be evoked were small: in the case of the deep mesencephalic nucleus (NMP, Fig. 2B, slice \#301) the rostro-caudal extension was $300-400 \mu \mathrm{m}$ and $200-300 \mu \mathrm{m}$ for the region medial to the rostral part of the dorsal lateral lemniscus (DNLL) (slice \#325 in Fig. 2C). Whereas the NMP cannot be clearly distinguished from neighboring structures, the area close to the DNLL is a distinct aggregation of large neurons.

\section{Types of elicited vocalizations}

Vocalizations resembling species-specific vocalizations. At most stimulation sites where vocalizations were elicited at low threshold currents, the spectral and temporal pattern of vocalizations corresponded to that of natural species-specific echolocation calls. Figure 3 shows a typical example of vocalizations elicited at a stimulus current of $8 \mu \mathrm{A}$. The left portion of the recording shows a stimulation run of about 7 seconds at low paper speed in which the individual echolocation calls are not resolved, whereas the larger right portion shows a temporally expanded sequence revealing the details of the frequency (second trace, F-VOC) and the intensity (third trace, I-VOC) of the calls. In the top trace (ELS) the onset of the stimulus train is represented by the falling edge of the pulses. In the example, the onset of vocalization shows a latency of about $50 \mathrm{msec}$. coinciding exactly with the onset of expiration (bottom trace, RESP). The respiration signal from the anemometer gives a qualitative measure of the temporal occurrence of the respiration.

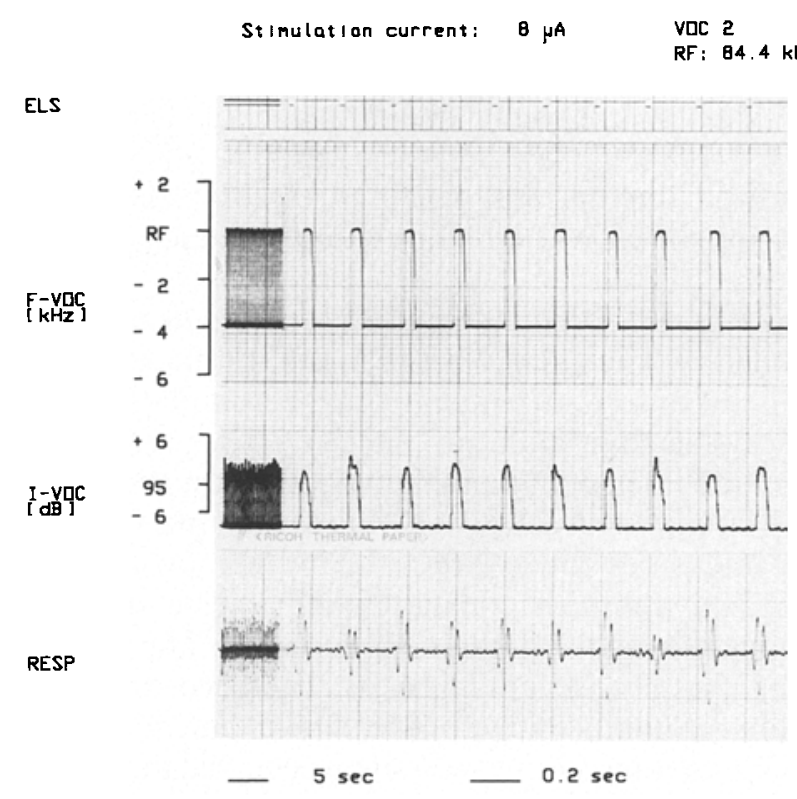

Fig. 3. Electrically elicited vocalizations typical for vocalization areas with low threshold currents. Lpper trace (EI.S): electrical stimulation bursts with negative going starting edge, the single electrical pulses are not resolved; second trace (F-VOC): frequency of the elicited vocalizations, only the frequency of the constant frequency portion is represented; third trace (I-VOC): envelope of the elicited vocalizations representing the instantaneous RMS-values of the waveforms; lower trace (RESP): qualitative representation of the respiratory activity, the first deviation marks the onset of expiration. Latency of vocalizations is around $30 \mathrm{~ms}$. that of respiration is around $20 \mathrm{~ms}$

For each respiratory cycle, the first fast deviation from background noise indicates the onset of expiration.

The frequency of the constant frequency portion $(\mathrm{CF})$, of the pulse (shown by the dark horizontal line at the top) is maintained at the individual resting frequency ( $R F$ ) of the bat during the entire stimulation sequence. Stimulation sequences contained typically more than 100 consecutive vocalizations, and the deviations of the frequency of individual calls corresponded to that of calls in regular, spontaneously emitted sequences. For individual bats the elicited vocalizations showed the same CF-frequency and typical deviations for all three vocalization specific areas. The frequency of the first echolocation pulses following the start of a stimulation sequence was sometimes slightly lower than the resting frequency (maximally $500 \mathrm{~Hz}$ ), but the frequency increased rapidly within a short series of pulses (2-5) to the resting frequency. This increase of $\mathrm{CF}$ at the start of call sequences is also found in spontaneously vocalizing bats. The duration of the calls was typically $40 \mathrm{msec}$. Visual inspection of the frequency-tovoltage converter signal at lower resolution showed 
that also the final frequency modulated portion was normal in electrically elicited vocalizations.

The typical sound pressure level of the emitted calls (I-VOC) lay between 95 and $105 \mathrm{~dB}$ and showed fluctuations which rarely exceeded $6 \mathrm{~dB}$ during an individual stimulation sequence.

Influence of stimulus intensity on vocalization parameters. The latency between the stimulus and vocal response is dependent on the stimulation site, and at each stimulation site, is a function of the applied intensity. Near the vocalization threshold, the latency is usually long $(40-80 \mathrm{~ms})$ and variable. At intensities above threshold, the latency stabilizes and stays almost constant over a wide range of suprathreshold stimulation currents. The latency was most consistent in areas where vocalizations were optimally elicited. The shortest mean latency (30.9 $\pm 9.1 \mathrm{~ms}$ s.d., $N=36$ sites) occurred in the area medial to the rostral DNLL. In the NMP the mean latency was $38.3 \mathrm{msec}$ (SD: $11.9 \mathrm{msec}, N=44$ ). The largest mean latency was measured in the deep layers and ventral parts of the intermediate layers of the SC at $47.0 \mathrm{msec}$ (SD: $22.2 \mathrm{msec}, N=103$ ). Within the SCi and SCd the latency of vocalizations was generally lower at more dorsal and posterior sites. Figure 4 compares the latency of vocalization from two stimulation sites with one penetration; the latency was $40 \mu$ s at a depth of $600 \mu \mathrm{m}$ and $80 \mathrm{~ms}$ at a depth of $100 \mu \mathrm{m}$. There was an abrupt transition of latency between these two stimulation sites. Despite the difference in latency from stimulus to vocal response, the latency from stimulus to expiration was the same at about $40 \mathrm{~ms}$ at the two locations. Respiration pulses synchronized by the stimulus showed a consistent latency of roughly $40 \mathrm{~ms}$ in all the investigated brain areas. The frequency and the sound pressure level of the elicited calls were constant over a wide suprathreshold range of stimulation currents at most sites where vocalizations were consistently elicited. With few exceptions, it was not possible to influence systematically the frequency or sound pressure level of the emitted vocalizations by changing the stimulus strength. The electrical stimuli acted as mere trigger signals for the emission of orientation calls.

The few stimulation sites where the sound pressure level of the elicited vocalizations changed systematically with stimulus intensity were concentrated in the caudal SCd. Figure 5 shows a sequence of vocalizations at increasing stimulus currents from 15 to $50 \mu \mathrm{A}$. As stimulus intensity is increased the frequency of the CF portion is not affected, but the sound pressure level of the echolocation calls rises. This rise in amplitude of vocalization from about 92 to $103 \mathrm{~dB}$ SPL is not accompanied by body movements or arousal of the animal. This feature is important, since stimulation currents high enough to arouse the animal can lead to an increase in sound pressure level of vocaliza-

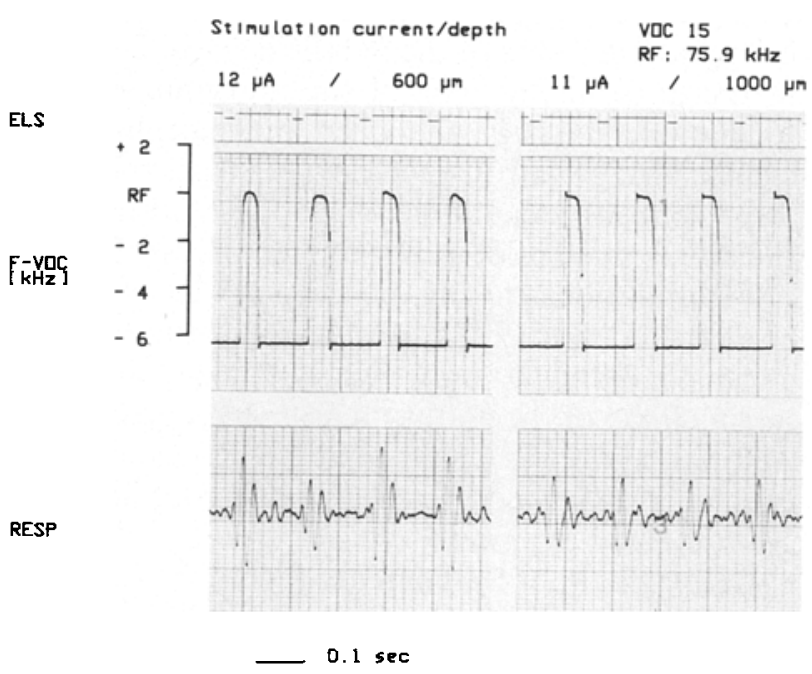

Fig. 4. Latency differences of elicited echolocation calls at two different depths in the deep layers of the superior colliculus. The latencies between electrical stimulation and vocalization were consistently at $40 \mathrm{~ms}$ around $600 \mu \mathrm{m}$ below the surface of the SC. About $400 \mu \mathrm{m}$ deeper, stimulation yielded echolocation pulses with latencies of $80 \mathrm{~ms}$ or above, whereas in both cases the latency of the expiratory pulse was consistently at $40-50 \mathrm{~ms}$

ELS

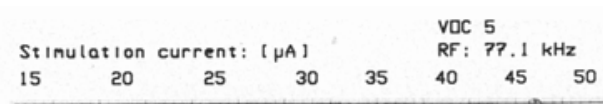

Fing
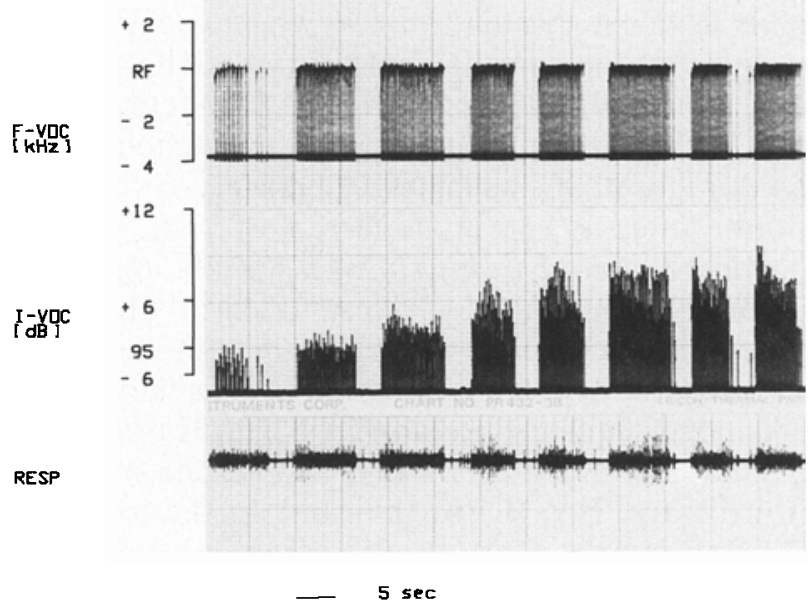

Fig. 5. Increase of sound pressure level of emitted orientation pulses with increasing stimulation current. Neither the frequency nor the duration of the pulses are affected by the stimulus current change. Above $35 \mu \mathrm{A}$ of stimulation current the sound pressure level reaches a saturation plateau of around $102 \mathrm{~dB}$ SPL. Stimulus site was within the caudal SCd 
tion, probably as a secondary effect. In rare cases, a change of the duration of the echolocation calls induced by the electrical stimulation in SCd was observed. With increasing stimulus current the duration of the calls increased without producing additional side effects like frequency or amplitude alterations or arousal.

Multiple vocalizations, i.e. double, triple or multiple calls during one expiration are rarely emitted spontaneously. In spontaneous sequences the rate of emission may rise but never leads to the formation of groups of pulses. By contrast, such grouped vocalizations were elicited by electrical stimulation, and the number of vocalizations evoked in each group was dependent on the stimulation current. Multiple vocalizations were only evoked consistently with currents at or above $20 \mu \mathrm{A}$, from the ventral edge of the rostral half of the CUN to about $500 \mu \mathrm{m}$ ventral to this nucleus. Figure 6 shows the formation of groups of vocalizations as a function of increasing stimulating currents $(50-110 \mu \mathrm{A})$. The sequences of pulses were emitted during one cycle of expiration and were closely grouped. In some cases, the intensity of the last pulses was somewhat reduced compared to the preceding pulses, which can be attributed to the fact that the available subglottic pressure builds up before and during the first pulses and is later reduced for subsequent pulses by prior pulse emission. Concurrently with the formation of grouped pulses, the mean sound pressure level of the emitted pulses is also increased. The CF-frequency, in con-

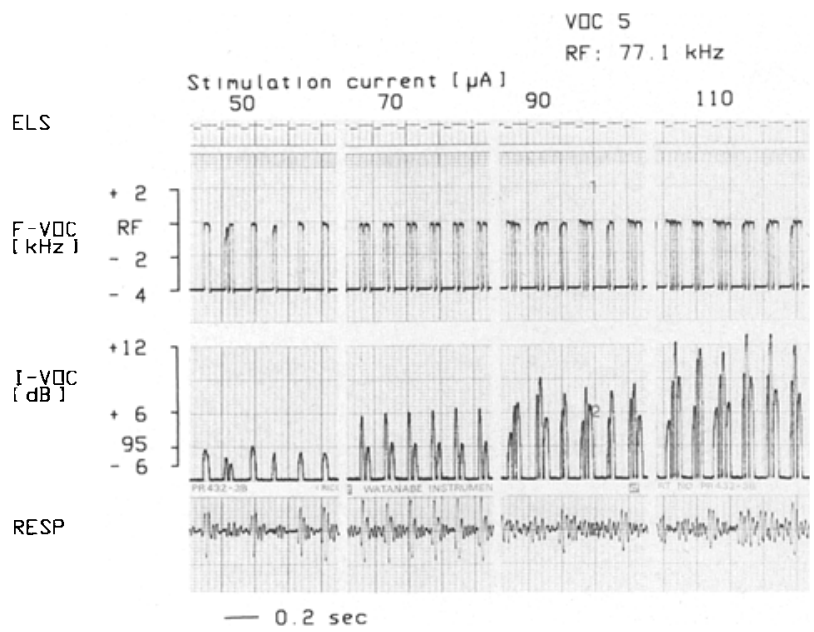

Fig. 6. Production of echolocation pulse trains. Increase of the stimulation current leads to the emission of bursts of up to 4 echolocation pulses. Concurrently the mean duration of the echolocation call bursts also increases with stimulus intensity $(36,60,72$ and $84 \mathrm{~ms}$ respectively). Stimulation site was situated ventrally to the rostral part of the cuneiform ncl trast, remains unchanged in single and in multiple pulse emissions.

Correlation between vocalization and respiration. At the brain sites where vocalizations were elicited (refer to Fig. 1), the respiratory cycle was closely synchronized to the electrical stimulus. The threshold current needed to produce synchronization of respiration was often lower than that needed to elicit vocalizations. Thus, respiration and vocalization were closely synchronized above vocal threshold. Apparently the stimulated structures influenced respiration and vocalization in a coordinated manner.

However, at some stimulation sites vocalization was elicited, but the respiratory cycle maintained its spontaneous rhythm. Depending on the stimulus repetition rate, a conflict between elicited vocalization and respiratory cycle could arise if the stimulation forced the emission of a vocalization immediately after an exhalation. This generally aroused the animal or caused a temporary deterioration in echolocation calls, mainly a drop in sound pressure level and/or a reduction of call duration. Figure 7 displays examples of correlated respiration and vocalization ( 2 examples at the left) and examples of conflicting respiratory rhythm and stimulation

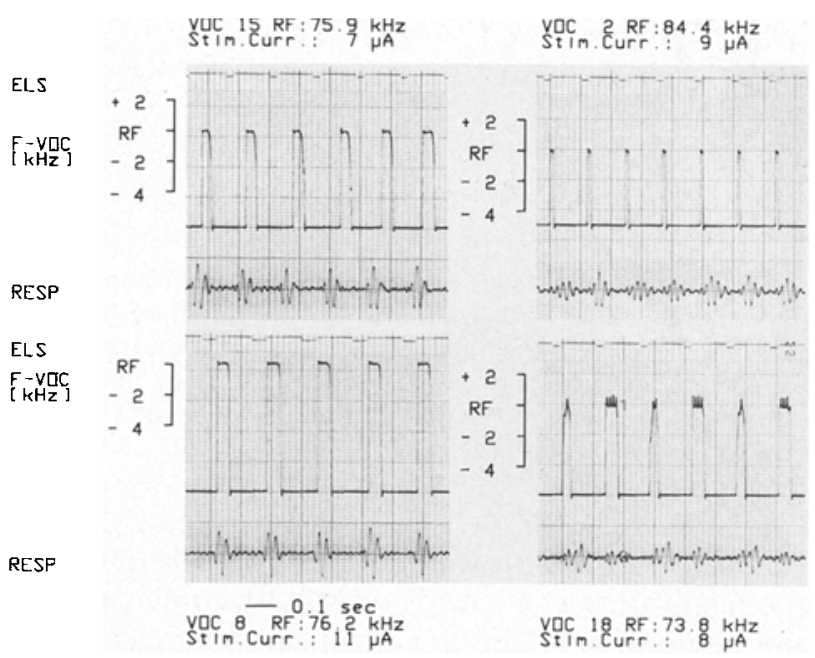

Fig. 7. Correlation of emitted echolocation calls and expiratory pulses. Left $:$ in both cases the expiratory pulses (latency around $40 \mathrm{~ms}$ ) are leading the emitted orientation calls, which themselves show different latencies (80 ms upper, 50-60 ms lower graph). The vocalizations do not show any distortions. This type of respiro-vocal correlation was found at stimulation sites as indicated in Fig. 1 (filled areas). Right: the expiratory pulses (latency around $40 \mathrm{~ms}$ ) lag behind the emission of the echolocation pulses with latencies of $20-30 \mathrm{~ms}$ (upper) and around $30 \mathrm{~ms}$ (lower). In both cases the vocalizations show distortions. This kind of respiro-vocal correlation is typically found at stimulation sites as indicated in figure 10 (single-hatched areas) 


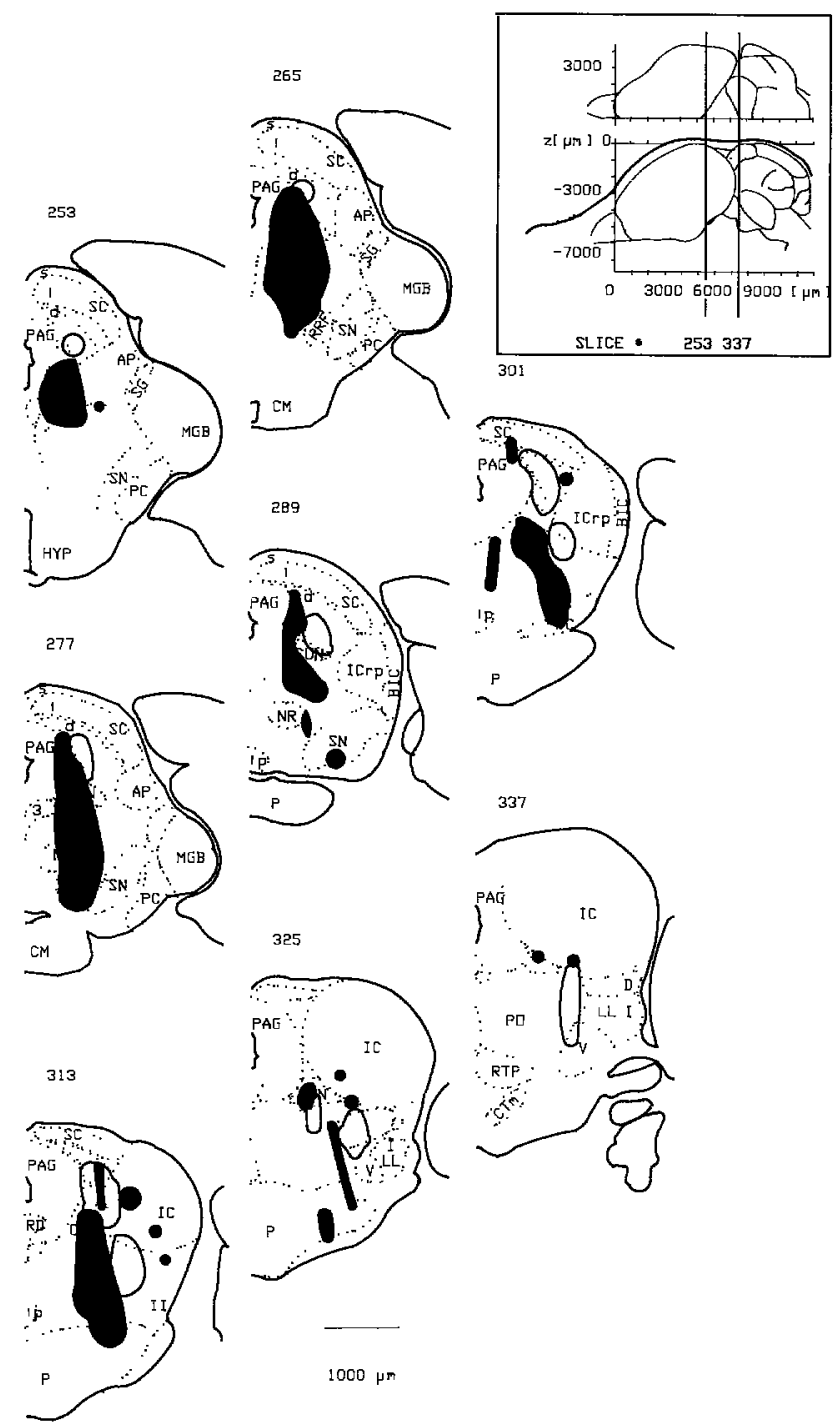

Fig. 8. Stimulation sites yielding synchronized respiratory activity without evoking vocalization at low threshold currents are shown as filled areas together with the optimal stimulation sites for eliciting of vocalizations (surrounded by heavy lines). The respiratory areas are mostly found medially and ventrally adjacent to the vocalization areas

rate ( 2 examples on the right). In the former cases the respiration cycle leads or exactly coincides with the beginning of the vocalization, whereas in the two latter examples the vocalization did not coincide with an early phase of expiration (upper right) or an extra expiratory pulse was generated by the electrical stimulus (lower right example) during emission of vocalization. If the vocalization and the respiration are not in register, the vocalizations commonly show very short durations and/or spectral distortions. Whereas the latency of the vocalizations shows considerable differences for the various examples ( $80 \mathrm{~ms}$ upper left, $50-60 \mathrm{~ms}$ lower left, 20-30 ms upper right and $30 \mathrm{~ms}$ lower right), the first peak of the respiratory pulse always appears with a similar latency of roughly $40 \mathrm{~ms}$ after the start of the stimulus burst.

Respiration could also be consistently synchronized to the stimulus at currents below $10 \mu \mathrm{A}$ in some areas where no vocalization could be elicited. These stimulation sites were situated just ventral and medial to locations where vocalization was electrically evoked and are shown in Fig. 8 as black areas.

Influence of repetition rate of the stimulus on sound emission. The rate of stimulation was an important parameter which, in many cases, determined the lowest threshold for eliciting vocalization, influenced the "quality" of the emitted calls and, if not appropriately chosen, led to arousal of the animal. The best repetition rate, i.e. with the least aversive effects on vocalization and behaviour of the bat, was generally slightly higher than the spontaneous respiratory rate $(3.5$ to $4 \mathrm{~Hz})$, or about double the respiratory rate (between 7 and $8 \mathrm{~Hz}$ ). Apparently, the small increase of the stimulus rate above the spontaneous respiratory rate leads to a stimulus induced synchronization of respiration without conflicting too much with the natural rhythm.

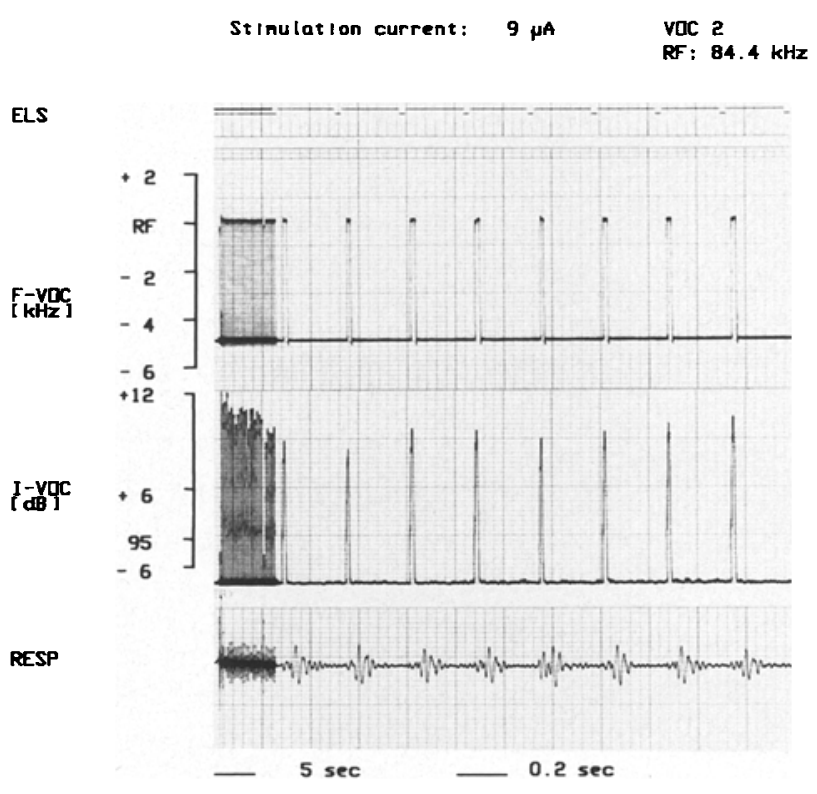

Fig. 9. Electrically elicited vocalizations showing distortions. The duration of the echolocation pulses is very short (smaller than $20 \mathrm{~ms}$ ) and the sound pressure level is higher than normal by 6 to $10 \mathrm{~dB}$, whereas the frequency of the constant frequency portion is at the resting frequency of the bat. No arousal or nonspecific body movements accompanied the utterances. It is noteworthy that the emission of the echolocation pulses preceded the expiratory pulse 
Distorted echolocation calls. At stimulation sites near the borders of vocalization areas and at other distinct sites (e.g. pontine regions) vocalizations deviated from the pattern of natural echolocation sounds at moderate stimulation currents (Fig. 10. single-hatched areas). These vocalizations were not accompanied by any body movements or arousal. Figure 9 gives a typical example of such distorted vocalizations. First, they showed unusually short durations of $20 \mathrm{msec}$ and below, relatively high sound pressure levels, but had normal latencies from stimulus onset of about $50 \mathrm{msec}$. Second.

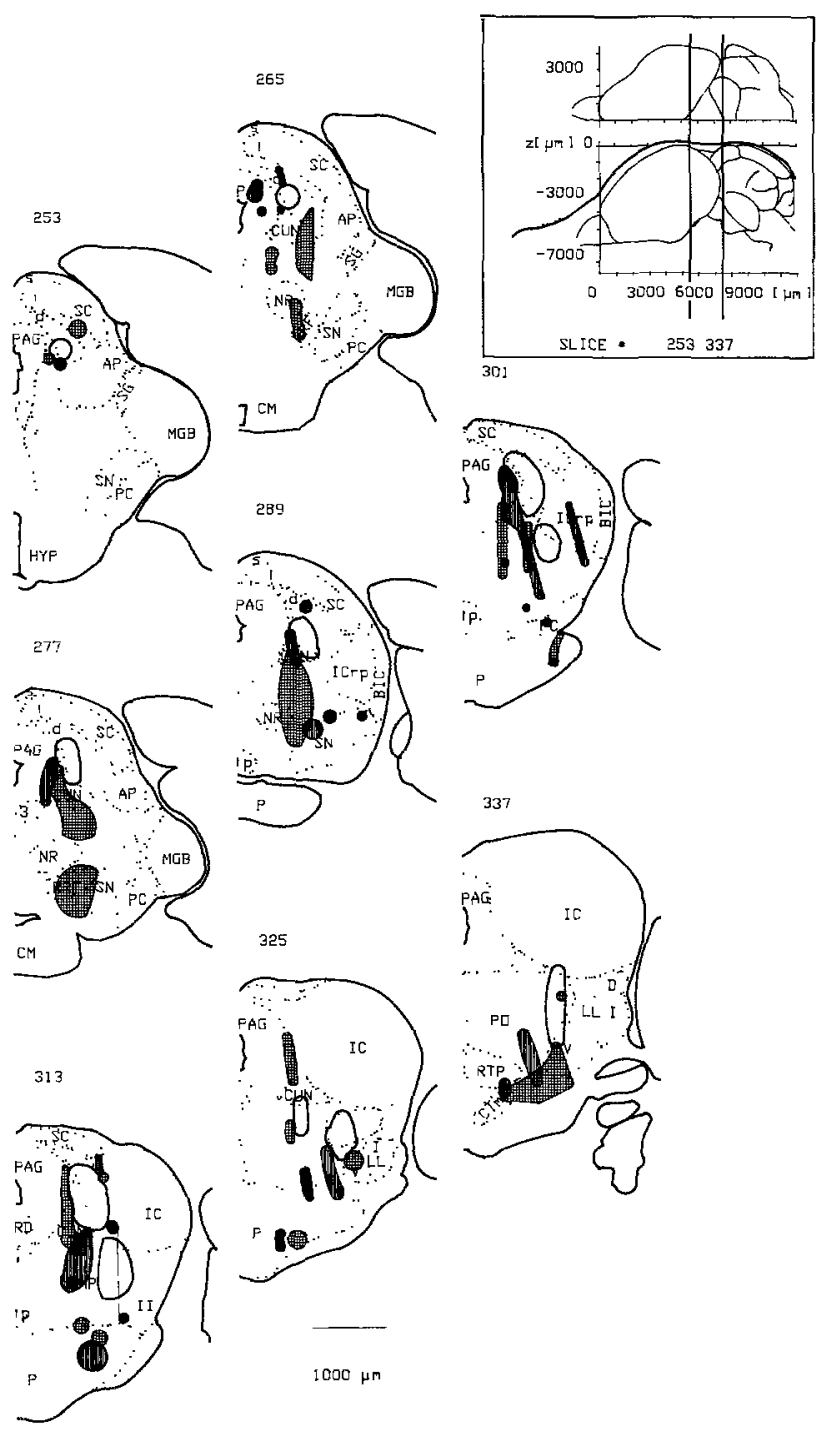

Fig. 10. Locations where vocalizations were distorted or accompanied by arousal of the animal. Stimulation sites yielding spectrally and/or temporally distorted vocalizations at low stimulation currents $(<20 \mu \mathrm{A})$ are represented by single-hatched areas. those yielding arousal of the animal are cross-hatched. For comparison, the regions where vocalizations were optimally elicited are surrounded by heavy lines there was in most cases a striking temporal discrepancy between the vocalization and the respiration signal. Although the respiration signal is tightly synchronized to the electrical stimulation it has a longer latency than the elicited vocalization. Thus the emission of the vocalization does not coincide with the expiratory cycle. This disruption of the coincidence between respiration and vocalization is probably the reason for the distortions. In a few cases the CF-frequency of the elicited calls fluctuated during stimulation sequences.

Spectro-temporal distortions of vocalizations also occurred together with body movements and/ or arousal of the animal. The cross-hatched areas in Fig. 10 demarcate locations at which electrical stimulation elicited vocalizations (mostly at thresholds above $20 \mu \mathrm{A}$, normal or distorted) together with arousal of the animal.

Vocalizations elicited in the lateral $P A G$ and the $C U N$. Although the thresholds for vocalizations were low at some positions in the lateral central gray matter (see Fig. 1, PAG), these vocalizations were always distorted and/or accompanied by various degrees of arousal of the animal. In the CUN, located ventrally adjacent to the deep SC (refer to Fig. 1), the vocalizations obtained at relatively low stimulus currents were normal, but the one-to-one relationship between stimulus and vocalization was disrupted and vocal responses were always accompanied by arousal. Thus, both structures (lateral central gray and cuneiform ncl.) are not considered specific for vocalization in the sense of the criteria described earlier.

\section{Discussion}

Echolocating horseshoe bats are well suited for studies of the vocalization system and the interconnections between the motor and the auditory systems. In few other animals does there appear to be as close a functional relationship between hearing and vocalization as in bats. The emission of echolocation sounds and the patterning of the calls are adapted to the demands of the hunting or orienting environment. Thus, information about the acoustical environment is transferred from the auditory system to the motor control system for vocalization. The structure of the echolocation calls is rather simple compared to communication signals in other mammals and therefore call control involves only relatively few parameters. The most striking example of a auditory-vocal interface is the Doppler-shift compensation of e.g. rhinolophid bats (Schnitzler 1968; Schuller et al. 1975). In this 
feedback system, the frequency of emitted pulse is lowered when, due to the Doppler-effect, echoes return with upward frequency shifts. This phenomenon is broadly independent of intensity and duration of the echolocation calls as long as they exceed a mimimum sound pressure level and a minimum duration (Schuller 1977).

In order to study the mechanisms of audiovocal interaction, the anatomical locations where such feedback could occur have to be determined. A precondition for investigating the descending vocalization system by electrical microstimulation methods in a small brain was the availability of an accurate stereotaxic system yielding spatial resolution down to about $100 \mu \mathrm{m}$, i.e. less than the dimensions of the investigated structures (minimum in the range of $200 \mu \mathrm{m}$ ). Previous electrical stimulation studies of vocalization in bats (Novick and Griffin 1961; Suga et al. 1973; Suthers and Fattu 1982), all lack accurate definition and verification of stimulation sites which would allow a precise attribution of distinct brain structures to the descending vocalization pathway.

\section{Technical considerations}

When electrical stimulation is used, it is often difficult to distinguish which neural elements, whether cells or fibers of passage, are actually excited. It is even more important to determine the extent of the region surrounding the electrode tip which is activated. Whereas for non-monopolar electrode arrangements no information on the exact distance to current relationship exists, the case of monopolar stimulation has been well investigated in different tissues, and reviewed by Ranck (1975). According to this review, stimulation currents of $10 \mu \mathrm{A}$ can activate myelinated fibers over a maximum distance of $180 \mu \mathrm{m}$ and neurons within a maximum distance of $120 \mu \mathrm{m}$ from the tip of the stimulating electrode. These distance-to-current relations are valid for monopolar, cathodic stimulus pulses of $0.2 \mathrm{~ms}$ duration. As the duration of stimuli used in this study was only half of that $(0.1 \mathrm{~ms})$, the effective radius for excitation was reduced by a factor of about 0.7 (120 and $80 \mu \mathrm{m}$ respectively). Therefore, the stimulation currents used in this paper yield a spatial resolution which parallels that reached by the stereotaxic procedure itself.

In order to define a stimulation site as specific for vocalization, several response criteria were used. We excluded regions in which the following responses not pertaining to vocalization behaviour were obtained: general arousal, body movements (except ear and/or oro-facial movements), and a disruption of the one-to-one relationship between electrical stimuli and vocalization. For the latter cases it was assumed that the stimulated elements were not directly involved in vocal control.

The requirement that the elicited vocalizations had to correspond in their spectral and temporal parameters to natural echolocation calls might appear overly restrictive. In reality, very few stimulation sites were found where this criterion was challenged, and in general, distorted vocalizations were accompanied by either arousal or body movements. The few regions where distorted vocalizations were evoked without body movement or arousal almost always lay at the border of vocalization-specific areas. Stimulation there generally provoked a mismatch of the phase between respiration and vocalization, so that the expiration led the vocalization. Since stimulation in the immediate neighborhood of sites which yielded "pathological" vocalizations induced a strong synchronization of respiration, it can be argued that predominantly respiratory neural elements were stimulated and that the distortions resulted from a respiratoryvocal mismatch. The longer-latency vocalization was forced into a period when inspiration occurs, whereas natural vocalizations are always emitted during the expiratory phase. A second possible mechanism by which alterations in the temporal or spectral pattern of vocalization could be induced depends upon the functional role of the stimulated structure. If the stimulated neurons were directly responsible for the laryngeal control of the temporal and spectral composition of the call by contributing to the input to the laryngeal muscles, then the electrical stimuli would disturb this coordination and lead to distorted vocalizations. Turning the argument around, this means that those stimulation sites which were termed "specific for vocalization" probably do not exert a direct influence on the spectro-temporal pattern of emitted orientation calls.

\section{Effect of stimulation on vocalization parameters}

The three vocalization-specific areas, namely, the ventral parts of SCi and the SCd, the NMP in the midbrain reticular formation, and the region medially adjacent to the rostral portion of the DNLL, mainly trigger vocalizations. This interpretation is further strengthened by the fact that in none of the three regions could a change of vocalization parameters be systematically induced by stimulation, with the exception of multiple call production and sound pressure level changes at a few loci. The latter is most probably produced by a stimulus 
intensity-dependent increase in expiratory flow, whereas the former is a typical timing effect involving the triggering of the multiple emissions.

It should be stressed that systematic changes in emission frequency could not be produced by stimulation in any of the sampled brain regions.

Electrical stimulation did not yield any functional correlation of brain areas with the Dopplershift control system. Nevertheless, it cannot be concluded from this finding that these regions are not involved in the frequency control of the emitted sounds. First, electrical stimulation might not be the right technique for producing differential influences on frequency-controlling neurons. Second, stimulation might only be effective if the bat is actively involved in a Doppler-shift compensation behaviour. Schuller (1986) showed that Dopplershift compensation was suppressed by electrical stimulation at some midbrain sites and the bat was forced to emit the resting frequency. Unfortunately no exact localization of the stimulation site is available.

Stimulation sites where rising stimulus intensity resulted in increasing sound pressure levels of emission most probably belong to the respiratory branch of the respiratory-vocal complex. The increase in the number of stimulated fibers or neurons most probably induced a stronger expiratory flow, which would ultimately lead to an increased subglottal pressure in the larynx as long as the vocal folds remain closed. The increased subglottal pressure consequently leads to a higher sound pressure level of the emitted echolocation calls. The frequency at any of the evoked sound pressure levels remained at resting frequency of the bat, meaning that at least in these structures, control of sound pressure level and frequency of the calls are not intimately interconnected.

Multiple echolocation calls, up to five within one burst, are also dependent on the strength of electrical stimulation. Interestingly, multiple vocalizations are elicited by short bursts $(15 \mathrm{~ms})$ of stimuli and are not a consequence of ongoing stimulation. The number of vocalizations within one burst is dependent on the intensity of stimulation and thus probably on the number of the stimulated neurons or fibers. The frequency is stable at the resting frequency of the bat throughout the individual bursts. Only variations of the sound pressure level of the emitted sounds accompany multiple vocalizations. Typically, the sound pressure level of the consecutive calls increases up to the second or third within one burst, and then declines to the end of the burst. This phenomenon may depend on the dynamics of expiratory flow and the maintenance of subglottal pressure throughout the burst. In the rising phase of the expiratory flow the closing of the glottis after the first pulse leads to a further increase of the subglottal pressure. The decrease of the subglottal pressure toward the end of the burst is probably due to the declining expiratory flow. The mechanism controlling the timing of the closure of the glottis is not accessible through experiments with electrical stimulation. The stimulation of multiple vocalizations as a function of stimulus intensity shows two phenomena: first, mechanisms leading to multiple vocalizations and mechanisms controlling sound pressure level, i.e. expiratory flow, are probably not completely functionally separated and, second, there must be a neural mechanism limiting the duration of single sounds within the bursts, thus determining the duration of the calls and also the silent periods between. In contrast to the intensity-dependent evocation of multiple calls, there was no clear effect of this parameter on the duration of the calls, although it was observed at a few stimulation sites.

\section{Anatomical substrates of vocal control}

Three areas where electrical microstimulation yielded vocalizations (and associated face and ear movements) which were identical to the natural echolocation sounds have been delimited. One of these areas, $\mathrm{SCd}$, is known to be an important center of sensori-motor coordination and integration (e.g. Huerta and Harting 1984). Some neurons within the deep layers display uni- or multimodal sensory responses, whereas others are involved in the control of motor orientation of body, head or ears. It is assumed that SCd is important for the guiding of orienting behaviour following sensory stimulation.

For echolocation, it is crucial that the orientation of the bat's head and ears in space be known during the emission of a highly directional sonar pulse. SCd might therefore be important for gating sound emission in synchrony with the orientation of head and ears. Consequently, it is not surprising that, in the SCd, spectral parameters of the vocalizations were not influenced by the electrical stimulation. Rather, this region had a largely triggering function for vocalization. Its influence on the timing of vocalizations is further supported by the finding that, at a few rostral sites, the temporal pattern of emission (multiple vocalizations) could be changed by electrical stimulation.

The second specific vocalization area appears homologous to the caudal part of the NMP in the rat (Paxinos and Watson 1986). This part of the 
tegmental gray is relatively undifferentiated and can be considered as a rostral continuation of the ventrolateral pontine reticular formation. It is bounded by the SC dorsally, the red nucleus and the PAG medially, the substantia nigra ventrally, and by the MGB laterally. In the bat, as in the rat, the nucleus has no clear boundaries with the surrounding tegmental structures and shows no distinct cytoarchitectonic pattern (Huber et al. 1943). In order to clarify the organization of this region the efferent and afferent connectivity of the NMP was studied by Veazey and Severin $(1982,1980 a, b)$ in the rat. No investigations on the functional involvement of the nucleus are available. Based on connectivity, the lateral NMP is considered to function in two separate systems. First, the lateral part, via connections to thalamic nuclei, may interact with the ascending activating system of the reticular formation. Second, the medial part, via its bulbar and spinal connections, may play a role in sensorimotor interactions (Veazey and Severin 1980a, b). Due to the undifferentiated morphology of this nucleus in the bat it is unclear whether a distinct subdivision of the NMP contained the responsive stimulation sites for vocalization. Only tracer injections in combination with prior stimulation experiments can reveal more details on the possible involvement of the DNM in the vocalization control pathway. The third specific vocalization site was found in a well-defined area rostral and medial to the DNLL. Electrical stimulation here elicited vocalizations with the greatest consistency, and were absolutely free of any accompanying elements not consistent with natural sound emission.

It is difficult to find a homology for this structure with a nucleus in other mammals. This has led to incorrect identification as the pedunculopontine nucleus in a preliminary report (Schuller and Radtke-Schuller 1988). Whereas its location and some connectional properties of this area support this homology, and it corresponds in many aspects to the pedunculopontine tegmental nucleus in the rat as described by Lee et al. (1988), the area is most probably not identical to the pedunculopontine nucleus as its neurons are not cholinergic (M. Vater, personal communication) as in other mammals. The area is sometimes designated as lateral tegmentum or paralemniscal zone (PLZ, e.g. Covey et al. 1987) because of its neighboring position to the lateral lemniscus, but it is, on the other hand, probably not identical with the paralemniscal zone in the cat as defined by Henkel and Edwards (1978) (PLZ lies more ventrally), who attribute to it a function in the control of pinna movements. The exact relationship between the vocalization-specific area described in this paper and the paralemniscal nucleus of Henkel and Edwards (1978) remains to be established and can only be defined by comparing the connectional patterns of the two areas (paralemniscal zone: Henkel 1981). From a functional point of view it seems very reasonable that pinna movements and timing of vocalizations are coordinated and that the activity of both areas contribute to this coordination. The coordination during vocalization of the head position, which determines the spatial emission pattern of the echolocation sound, and the position of the pinnae, which determines the spatial pattern of sound reception, is necessary for reliable spatial orientation with echolocation sounds. In the three regions so far discussed, electrical stimulation did not interfere with the spectral or temporal pattern of echolocation calls, but mainly triggered the vocalization and some oro-facial motor patterns commonly accompanying echolocation behaviour. These regions can therefore be termed "premotor" areas which do not directly influence the final motor pattern of echolocation call emission.

There were two other regions which yielded echolocation calls upon electrical stimulation, namely, the CUN and lateral parts of the pontine gray. Stimulation in the pontine gray always led to distortions of the spectro-temporal pattern of vocalization, possibly caused either by activating brain structures directly involved in the vocal control pathway or by interfering with areas having respiratory control functions. Rübsamen and Schweizer (1986) demonstrated that neurons in the lateral pontine gray projected to rostral portions of the motor nucleus of the larynx (ncl. ambiguus). Thus, the stimulation of the pontine areas potentially influences the vocal pattern via the connections to the nucleus ambiguus.

Stimulation of the CUN elicited vocalization in a more general sense, i.e. the one-to-one relationship between stimulus and response was disrupted and the vocal utterances occurred as a consequence of prior electrical stimulation. The spectrotemporal pattern of vocalization was not disturbed, but the elicited vocalizations were accompanied by arousal of the animal even at low stimulation currents. The CUN and the adjacent lateral periaqueductal gray are also sources of direct projections to the ncl. ambiguus (Rübsamen and Schweizer 1986; Schuller and Radtke-Schuller 1988). The PAG in primates is considered to be an important relay of the descending vocalization system, mediating all input from higher brain levels finally to the nucleus ambiguus. No distinction is made in the primate 
studies between the PAG and the immediately adjacent cuneiform nucleus, so that the obligatory relay function for vocalization in primates may also involve this nucleus. This region receives (among other projections) input from the hypothalamic areas involved in emotional influences on vocalization (Jürgens and Ploog 1981). The arousing effect of electrical stimulation in this area could be explained on this connectional basis.

An understanding of the differential role of these brain areas in the control of vocalization should result from recordings and detailed anatomical investigations.

Acknowledgements. We thank G. Neuweiler, W.E. O'Neill and J. Wenstrup for their comments on this manuscript and the correction of the English version. We also thank G. Franke and Miss H. Pötke for technical assistance. Supported by the Deutsche Forschungsgemeinschaft, Sonderforschungsbereich "Gehör" (204/TP10)

\section{References}

Covey E. Hall WC, Kobler JB (1987) Subcortical connections of the superior colliculus in the mustache bat, Pteronotus parnellii. J Comp Neurol 263:179-197

Gooler DM, O'Neill WE (1987) Topographic representation of vocal frequency demonstrated by microstimulation of anterior cingulate cortex in the echolocating bat, Pteronotus pamelli parnelli. J Comp Physiol [A] 161:283-294

Henkel CK (1981) Afferent sources of the lateral midbrain tegmental zone associated with the pinnae in the cat as mapped by retrograde transport of horseradish peroxidase. J Comp Neurol 203:213-226

Henkel CK, Edwards SB (1978) The superior colliculus control of pinna movements in the cat: possible anatomical connections. J Comp Neurol 182: 763-776

Huber GC, Crosby EC, Woodburne RT, Gillilan LA, Brown LO, Tamthai B (1943) The mammalian midbrain and isthmus regions. I. The nuclear pattern. J Comp Neurol 78: $129-534$

Huerta MF, Harting JK (1984) The mammalian superior colliculus: studies of its morphology and connections. In: Vanegas (ed) Comparative neurology of the optic tectum. Plenum Press, New York, pp 687-773

Jürgens U (1976) Reinforcing concomitants of electrically elicited vocalizations. Exp Brain Res 26:203-214

Jürgens U (1988) Central control of monkey calls. In; Todt D, Goedeking P. Symmes D (eds) Primate vocal communication. Springer, Berlin Heidelberg New York Tokyo, pp $162-167$

Jürgens U, Hast M, Pratt R (1978) Effects of laryngeal nerve transection on squirrel monkey calls. J Comp Physiol $123: 23-29$

Jürgens U. Ploog D (1981) On the neural control of mammalian vocalization. TINS 6:135-137

Jürgens U. Pratt R (1979) Role of the periaqueductal grey in vocal expression of emotion. Brain Res 167:367-378

Jürgens U, Richter K (1986) Glutamate-induced vocalization in the squirrel monkey. Brain Res 373:349-358

Kirzinger A. Jürgens U (1985) The effects of brainstem lesion on vocalization in the squirtel monkey. Brain Res $358: 150-162$
Lee HJ, Rye DB, Hallanger AE, Levey AI. Wainer BH (1988) Cholinergic vs. noncholinergic efferents from the mesopontine tegmentum to the extrapyramidal motor system nuclei. J Comp Neurol 275:469-492

Müller-Preuss P. Ploog D (1983) Central control of sound production in mammals. In: Lewis $B$ (ed) Bioacoustics. Academia Press, London, pp 125-146

Novick A, Griftin DR (1961) Laryngeal mechanisms in bats for the production of orientation sounds. J Exp Zool $148: 125-145$

Paxinos G. Watson $\mathrm{Ch}$ (1986) The rat brain in stereotaxic coordinates, 2nd edn. Academic Press, New York

Ploog D (1988) Neurobiology and pathology of subhuman vocal communication and human speech. In: Todt D, Goedeking P. Symmes D (eds) Primate rocal communication. Springer, Berlin Heidelberg New York Tokyo, pp 195-212

Ranck JB (1975) Which elements are exited in electrical stimulation of mammalian central nervous system: a review. Brain Res 98:417-440

Richter K, Jürgens U (1986) A comparative study on the elicitability of vocalization by electrical brain stimulation, glutamate. asparate and quisqualate in the squirrel monkey. Neurosci Lett 66:239-244

Rübsamen R, Betz M (1986) Control of echolocation pulses by neurons of the nucleus ambiguus in the rufous horseshoe bat. Rhinolophus rouxi. I. Single unit recordings in the motor nucleus of the larynx in activity vocalizing bats. J Comp Physiol [A] 159:675-687

Rübsamen R, Schuller G (1981) Laryngeal nerve activity during pulse emission in the CF-FM bat, Rhinolophus ferrumequinumn. II. The recurrent laryngeal nerve. J Comp Physiol 143:323-327

Rübsamen R, Schweizer H (1986) Control of echolocation pulses by neurons of the nucleus ambiguus in the rufous horseshoe bat, Rhinolophus rouxi. II. Afferent and efferent connections of the motor nucleus of the laryngeal nerves. J Comp Physiol [A] 159:689-699

Schnitzler HU (1968) Die Ultraschall-Ortungslaute der Hufeisen-Fledermäuse (Chiroptera-rhinolophidac) in verschiedenen Orientierungssituationen. Z Vrgl Physiol 57:376-408

Schuller G (1977) Echo delay and overlap with emitted orientation sounds and Doppler-shift compensation in the bat, Rhinolophus ferrumequinum. J Comp Physiol 114:103-114

Schuller G (1980) Hearing characteristics and Doppler-shift compensation in South Indian CF-FM bats. J Comp Physiol 139:349-356

Schuller G (1986) Influence of echolocation pulse rate on Doppler-shift compensation control system in the greater horse-shoe bat. J Comp Physiol [A] 158:239-246

Schuller G, Radtke-Schuller S (1988) Midbrain areas as candidates for audio-vocal interface in echolocating bats. In: Nachtigall PE (ed) Animal sonar systems. Helsingor symposium. Plenum Press, New York

Schuller G, Rübsamen R (1981) Laryngeal nerve activity during pulse emission in the CF-FM bat, Rhinolophus ferrumequinilin. J Comp Physiol 143:317-321

Schuller G. Suga N (1976) Laryngeal mechanisms for the emission of CF-FM sounds in the Doppler-shift compensating bat, Rhinolophus ferrumequinum. J Comp Physiol 107:253-262

Schuller G, Beuter K, Schnitzler H-U (1974) Response to frequency shifted artifical echoes in the bat. Rhinolophus ferrumequinum. J Comp Physiol 89:275-286

Schuller G, Beuter K, Rübsamen R (1975) Dynamic properties of the compensation system for Doppler-shifts in the bat, Rhinolophus ferrumequinum. J Comp Physiol 97: 113-125 
Schuller G, Radtke-Schuller S, Betz M (1986) A stereotaxic method for small animals using experimentally determined reference profiles. J Neurosci Methods 18:339-350

Schweizer H, Rübsamen R, Ruehle C (1981) Localization of brainstem motoneurons innervating the laryngeal muscles in the rufous horseshoe bat, Rhinolophus rouxi. Brain Res $230: 41-50$

Suga N, Schlegel P, Shimozawa T, Simmons J (1973) Orientation sounds evoked from echolocating bats by electrical stimulation of the brain. J Acoust Soc Am 54:793-797

Suthers RA, Fattu JM (1982) Selective laryngeal neurotomy and the control of phonation by the echolocating bat, Eptesicus. J Comp Physiol 145:529-537

Thoms G, Jürgens U (1981) Role of internal laryngeal nerve in phonation: an experimental study in the squirrel monkey. Exp Neurol 74: 187-203
Thoms G, Jürgens U (1987) Common input of the cranial motor nuclei involved in phonation in squirrel monkey. Exp Neurol 95:85-99

Veazey RB, Severin CM (1980a) Efferent projections of the deep mesencephalic nucleus (pars lateralis) in the rat. J Comp Neurol 190:231-244

Veazey RB, Severin CM (1980b) Efferent projections of the deep mesencephalic nucleus (pars medialis) in the rat. J Comp Neurol 190:245-258

Veazey RB. Severin CM (1982) Afferent projections to the deep mesencephalic nucleus in the rat. J Comp Neurol 204: 134-150

Received March 16, 1989 / Accepted September 19, 1989 